\title{
Cancer treatment: deciding what we can afford
}

\author{
Finding out what the money buys is the first step
}

Most patients with lung cancer either present with or develop advanced disease that treatment with surgery or radiotherapy will not cure. Non-small cell tumours ( $80 \%$ of the total) respond poorly to chemotherapy - about a third regress for a few months-and a recent consensus conference recommended that chemotherapy for these tumours should not be given outside clinical trials. ' For haemoptysis and bone pain palliation can usually be obtained by single treatments or short courses of radiotherapy. How resources are used in treating this neoplasm, the commonest malignant cause of death, is germane to the treatment of incurable cancer.

A Canadian randomised trial compared two regimens of chemotherapy containing cisplatin (CAP and VP) with a conservative policy of "best supportive care" for patients with advanced non-small cell lung cancer. ${ }^{2}$ All patients were given palliative radiotherapy as required. Patients receiving best supportive care survived for a mean of 24 weeks. Mean survival benefits of eight and 13 weeks were achieved with CAP and VP respectively. Do these benefits justify the toxicity and financial cost of chemotherapy? Is expenditure on such treatment compatible with a fair distribution of NHS money?

This trial incorporated an economic evaluation. At 1991 prices best supportive care costs on average about $£ 6000$ for each patient. The CAP regimen saved about $£ 700$ because those receiving best supportive care spent substantially more time in hospital. In contrast, the most effective VP regimen was associated with an additional cost of about $£ 2500$ (or $£ 10000$ per year of life gained) because the shorter time spent in hospital was not enough to offset the cost of chemotherapy.

This study shows some of the many complexities of economic evaluation. It was conducted before the introduction of generic cisplatin, and the additional cost of VP would be reduced by a third at present drug prices. The costs of hospital stays were substantially greater than drug costs, however, making them the most influential component of the total. While such toxic regimens usually require inpatient administration, the need for hospital admission for patients not receiving such treatment is not defined. For such patients to spend 24 days in hospital would be unusual in Britain.

The benefits of cancer treatment are longer survival, relief of symptoms, or both. Deciding whether a patient has benefited overall from a treatment is often difficult, especially if the treatment was very unpleasant. The cost for each year of benefit of potentially curative treatments, however, is usually not much higher than $£ 1000$ per patient. ${ }^{3}$ Although some palliative treatments - for example, cheap hormonal drugs for breast and prostate carcinoma and single fractions or very short outpatient courses of radiotherapy for bone pain and haemoptysis - cost less than $£ 1000$ for each year of benefit; palliative chemotherapy and more prolonged regimens of radiotherapy, particularly if given to inpatients or those using ambulance transport, may cost several thousand pounds per year of benefit. Adjusting for quality of life may be invidious as an observer's assessment of quality may correlate poorly with the patient's assessment of value. If this is done, however, the difference in costs for each year of benefit between palliative and potentially curative treatments is even greater.

Adjuvant chemotherapy for potentially curable breast cancer patients with presumed but undetectable micrometastases may improve long term survival. ${ }^{+}$It has also been vindicated by the duration without symptoms and treatment toxicity, adjusted for quality of life. ${ }^{5}$ In many hospitals this treatment is not offered, however, while chemotherapy for those with overt metastatic disease is readily available. Although only about one in 10 additional premenopausal patients who are node positive enjoys long term survival from adjuvant chemotherapy, the cost for each year of benefit is still probably less than $£ 1000$.

Unrealistic hopes of benefit may lead easily to substantial resources being committed to anticancer treatments for patients who are terminally ill. ${ }^{67}$ Some purchasing authorities may feel that adjuvant chemotherapy for potentially curable patients is a better buy. They may also be influenced by variations in the cost of potentially curative treatments. In one comparison of oesophagectomy and radiotherapy for oesophageal carcinoma the median survival was identical, but the cost of surgery was four times higher because of intensive perioperative care and prolonged hospital stays for those with complications. ${ }^{8}$ Savings made by stopping treatments that are poor value for money could be used to increase consultation time, hospice care, and counselling.

In 1985 Professor Roberts and his colleagues in Cardiff estimated that the NHS could not afford more than $£ 14000$ to save a life.' (This would now be about $£ 20000$.) They concluded that breast cancer screening at $£ 80000$ for each life saved was an inequitable use of resources. This is comparable with the Forrest report's estimate of $£ 3000$ for each year of life saved. ${ }^{10}$

In Britain in 1991 treatments costing $£ 1000$ for each year of 
benefit-whether this is an improvement in quantity or quality - seem to be excellent value. Those costing $£ 10000$ for each year of benefit probably represent an unfair distribution of resources - a verdict likely to apply to many treatments costing between $£ 1000$ and $£ 10000$ for each year of benefit. All treatments of debatable value need economic as well as thorough clinical assessment, and oncologists and their colleagues in the laboratory need to develop better ways of predicting which patients will benefit from them.

Consultant in Clinical Oncology,

GARETH J G REES

Royal United Hospital,

Bath BAl 3NG
Timothy AR, Ash D, Corris $\mathrm{P}$, et al. Workshop on consensus guidelines for management of lung ancer. Clin Oncol 1990;2:97-101.

Jaakkimainen L, Goodwin PJ, Pater J, Warde P, Murray N, Rapp E. Counting the costs of chemotherapy in a National Cancer Institute of Canada randomized trial in non small-cell lung cancer. F Clin Oncol 1990;8:1301-9.

Rees GJG. Cost-effectiveness in oncology, Lancet 1985;ii: 1405-8.

4 Early Breast Cancer Trialists' Collaborative Group. Effects of adjuvant tamoxifen and of cytotoxic chemotherapy on mortality in early breast cancer. An overview of 61 randomized trials among chemotherapy on mortality in early breast cancer.

Goldhirsch A, Gelber RD, Simes RJ, Glasziou P, Coates AS. Costs and benefits of adjuvant therapy in breast cancer: a quality-adjusted survival analysis. 7 Clin Oncol 1989;7:36-4.

6 Jennett $B$, Buxton $M$. When is treatment for cancer economically justified? $\mathcal{F} R$ Soc Med 1990);83:25-8. (Discussion paper.)

7 Holli K, Hakama M. Treatment of the terminal stages of breast cancer. BMJ 1989;298:13-4.

8 Walker QJ, Salkeld G, Hall J, et al. The management of oesophageal carcinoma: radiotherapy or surgery? Cost considerations. Eur f Cancer Clin Oncol 1989;25: 1657-62.

9 Roberts CJ, Farrow SC, Charny MC. How much can the NHS afford to spend to save a life or avoid a severe disability? Lancet $1985 ; \mathrm{i}: 89-91$.

10 Working Group. Breast cancer screening. Report to the Health Ministers of England, Wales, Scotland. and Northern Ireland. London: HMSO, 1986. (Chairman Professor Sir Patrick Forrest.)

\section{In search of consensus}

\section{No agreement on who should write guidelines or how they should be used}

Almost every month-or so it seems-a new consensus statement or set of guidelines is published attempting to specify the optimum management of some common condition. Some are drawn up by prestigious organisations such as the United States National Institutes of Health, the Rand Corporation, or the Royal College of Physicians while others emerge from ad hoc meetings by small self selected groups. In this world of consensus there is, however, no agreement on how guidelines should be created or by whom, nor on how doctors should be expected to react to them when they appear.

These questions were tackled at a recent meeting on expert groups organised by the King Edward's Hospital Fund for London. The common ground agreed by the participants was that there is good evidence that patients treated according to a defined protocol generally do better than those treated freehand by an individual doctor. How, then, should the protocols be established.

The first task, clearly, is for an individual or groups to review the relevant published material to ascertain what (if anything) has been established by well designed, properly analysed research studies. For most topics the published reports are, in the words of one expert at the meeting, "rich but often inappropriate." In the Rand Corporation model an exhaustive literature review is followed by filling in the gaps by discussions with clinicians. The organisers then construct a list of all the clinical features of potential patients for, say, coronary angioplasty (age, sex, blood pressure, history, features of the electrocardiogram at rest and during exercise, etc). A consensus panel of clinicians then looks at these indications in terms of clinical risks and benefits using first a Delphi procedure in which each participant scores the indications and then modifies his or her scores in the light of scores recorded by the others, and then a discussion session lasting one and a half days. At the end of this expensive and time consuming process the clinical indications are each scored on a rating of one to nine. The King's Fund meeting was told that when results obtained in this way are presented to clinicians who took no part in their preparation there is little disagreement about what care is appropriate for a patient with a particular clinical profile-though there might be less agreement about whether or not the care was necessary.

The Rand method puts the clinical indications under a microscope but does not attempt any judgments about costs or social priorities. At the National Institutes of Health and at the King's Fund consensus meetings the approach has differed in two ways: the published data have been reviewed using individual experts' assessments rather than a complete literature review, and the consensus panels have included non-medical experts and lay people and have tried to look at wider issues such as the affordability of a procedure and how it should be measured against other competing demands for medical resources.

During discussion all agreed that however a meeting to create guidelines was structured the clinicians who took part found the experience worthwhile as they began questioning their accepted patterns of practice and justifying them to colleagues. There was strong support, too, for having a chairman who, while medical (and thus familiar with the language and the concepts), had no special knowledge of the topic being examined. Ideally, the expert panel should separate what was reasonably certain from what was no more than informed opinion and should also identify the research topics that needed urgent attention.

Is it a good idea for small, local groups to attempt to produce guidelines of this kind? At very least the exercise itself may be expected to be both enjoyable and educational, and there is plenty of evidence that clinicians are much more likely to follow guidelines if they have played some part in creating them. Nevertheless, the King's Fund meeting seemed persuaded that it was a waste of time and money for hundreds of collections of doctors to meet to devise protocols for treating conditions such as hypertension or diabetes when so many good sets of guidelines already exist. What the meeting wanted to see was a few representative, national bodies drawing up basic guidelines: these could then be distributed around the country to local groups which could then discuss them, modify them to take account of local cultural and social features, and in so doing give the local clinicians the essential feeling of ownership of the management protocols.

And beyond treatment protocols? At the edge of the map there may be dragons - the whole new vista of assessing the outcome of treatment, measuring the effects of introducing a protocol, modifying it to take account of the findings, measuring the outcomes of the modified protocol-truly a process that will never (and should never) be completed.

Associate editor, $B M \mathcal{F}$ 